\title{
Green Synthesis of Silver Nanoparticles with High Fungicidal Activity from Olive Seed Extract
}

\author{
Habeeb Khadri ${ }^{1}$, Mohammad Alzohairy ${ }^{1}$, Avilala Janardhan², \\ Arthala Praveen Kumar ${ }^{2}$, Golla Narasimha ${ }^{2 *}$ \\ ${ }^{1}$ Department of Medical Laboratory, College of Applied Medical Sciences, Qassim University, Qassim, Kingdom of Saudi Arabia \\ ${ }^{2}$ Applied Microbiology Laboratories, Department of Virology, Sri Venkateswara University, Tirupati, India \\ Email:"gnsimha123@rediffmail.com
}

Received May 23, 2013; revised June 23, 2013; accepted July 1, 2013

Copyright (C) 2013 Habeeb Khadri et al. This is an open access article distributed under the Creative Commons Attribution License, which permits unrestricted use, distribution, and reproduction in any medium, provided the original work is properly cited.

\begin{abstract}
Silver nanoparticles in the form of silver based chemicals trace back their origin to time immemorial since the dilute forms of silver nitrate were used in place of antibiotics before they dominated the field of medicine. But, it has now become necessary to explore the anti-microbial properties of silver based chemicals again due to the microbes gaining resistance against the wide range of present day antibiotics. The advancements in the field of medicine and technology started to coalesce to combat the adaptability of microbes as they successfully become tolerant to antibiotics and it manifested in the form a current technology, Nanomedicine. Nanomedicine deals about the medicines at a nano scale to rarefy the intensity of medicine to unaffected tissues and reduce the volume of medicine used. In the present context, our attempt is to develop potential anti-microbial particles in the form of silver nanoparticles by using the biological phenomena which we call Green synthesis an eco-friendly approach to conventional chemical synthesis. The enzymatic machinery of the olive seeds has been exploited to produce silver nanoparticles and test their efficacy as antifungal agents before we characterized their physical properties using UV-Vis, TEM, and FTIR analysis. The efficacy of these particles as antagonists on fungal pathogen Aspergillus niger a causative agent of Aspergillosis in human beings and is promising and they have a lot of scope for the purpose and hope the technology leads the next generation of anti-microbials.
\end{abstract}

Keywords: Olive Seeds; Silver Nanoparticles; Characterization; Fungicidal Activity

\section{Introduction}

Nanotechnology is one of the most interesting and open inquisitive fields for research in modern material science. The nano-sized particles of routine materials are emerging surprisingly with new applications at a brisk pace. The conventional methods of synthesis of nanoparticles using chemical means disturb the tranquility of the ecosystem and pose a serious environmental threat and thus novel methods that address the ecological safety are to be explored for and also consequently they must increase quantitatively the output to benefit from the advantages of nanotechnology. Living systems are bequeathed with several chemical principles and hence the biomimetic approach of utilizing them could be a best alternative significantly reducing the usage of chemical reagents. Wide range of biological systems from bacteria to plants and mammals can be used for synthesizing nano-sized parti-

"Corresponding author. cles of interest. However, ethical constraints and the productivity force us towards the microbes and plants. Most important of them is synthesis using plant extracts eliminating the laborious process involving the maintenance of microbial cultures. Biological molecules as reducing agents and oxidizing agents are significantly safe over their chemical counterparts thus obviating the need of chemical reagents for synthesis. By taking the above aspects into consideration, biological sources and biomaterials are best alternative means by all means for the synthesis of nanoparticles. In recent years, various chemical methods have been replaced by biological for the synthesis of silver nanoparticles $[1,2]$. The natural products such as monosaccharide or plant extracts have been used as reducing agents during these studies. Microbiological methods are much slower than plant extracts and other chemical reducing agents for production of nanoparticles as observed from many studies. Commercial viability of the nanoparticles would be greater when they could be syn- 
thesized biologically and more rapidly in the reaction vessels. In general, all the biological systems are rich in reductase enzymes and olive plant is no exception, and they obviously carry the ability of reducing inorganic salts to their respective elemental forms and this mechanism has been exploited earlier and the present attempt is to use olive seeds for reducing silver nitrate salts to metallic silver and olives that have an advantage because of their inherent antimicrobial abilities. Sastry and colleagues [3] pioneered the use of plant extracts to synthesize nanoparticles and have reported syntheses of nanoparticles at rates on par with chemical reagents. Various metallic nanoparticles of Silver, Gold, Zinc, Palladium etc., are being synthesized using biological systems [4]. Uchida et al., 2003 [5], Kumar and Munsted [6] suggested that the antimicrobial activity of Ag may be due to the binding of the $\mathrm{Ag}^{+}$cation to electron donor groups in biological molecules containing sulfur, oxygen or nitrogen (e.g. enzymes) which, in turn, results in the loss of their function. Another explanation was put forward by Kim et al 2007 [7] is based on the generation of free radicals from the surface of the Ag-NPs which finally result in breakdown of bacterial membrane function. Khalil et al. (2001) [8] reported that due to the presence of many acids like, protocatechuic acid, coumaric acid, ferulic acid, cinnamic acid, oleuropein etc., the olive plant has excellent antifungal properties. Olive cakes have shown antifungal properties against several fungi like Fusarium, Rhizopus, Stemphylium, Rhizoctonia, Alternaria etc. In our current study, the silver nanoparticles have been synthesized using screened extracts of the olive plant seeds and report that silver nanoparticles can be applied effectively in the control of microorganisms and the prevention of deleterious infections. Our results support the hypothesis that $\mathrm{Ag}$ nanoparticles can be prepared in a simple and cost-effective manner and are suitable for formulation of new types of fungicidal agents. UV-Vis spectral analyses, Transmission electron microscopy (TEM), Fourier transform infrared spectroscopy (FTIR) analysis was used to characterize the silver nanoparticles formed.

\section{Experimental Details}

\subsection{Preparation of Dried Biomass}

The fresh olive plant seeds were exposed to the sun until they were completely dried. The extract used for the reduction was prepared by crushing the dried seeds and then screening the seed powder by a 20 mesh sieve.

\subsection{Biosynthesis of Silver Nanoparticles}

The olive plant seed extraction was prepared by boiling a mixture of $5 \mathrm{~g}$ of sieved dried seeds powder into $100 \mathrm{~mL}$ of sterile distilled water in an Erlenmeyer flask for $5 \mathrm{~min}$ and filtered through Whatman No.1 filter paper (pore size $25 \mu \mathrm{m})$. The filtrate was further filtered through 0.6 $\mu \mathrm{m}$ sized filters. The solution was decanted and stored at $4^{\circ} \mathrm{C}$; it was used within a week of its preparation. $1 \mathrm{mM}$ aqueous solution of silver nitrate $\left(\mathrm{AgNO}_{3}\right)$ was prepared and used for the synthesis of silver nanoparticles. $10 \mathrm{ml}$ of olive seed extract was added into $90 \mathrm{ml}$ of aqueous solution of $1 \mathrm{mM}$ silver nitrate for bioreduction of $\mathrm{Ag}^{+}$ ions in the solution and kept at room temperature for 24 hours.

\subsection{UV-Vis Spectrum Analysis}

The bioreduction of pure $\mathrm{Ag}^{+}$ions in aqueous solution was monitored by sampling of aliquots of suspension subsequently measured UV-Vis spectrum of the reaction medium at 24 hours after diluting a small aliquot of the sample into distilled water. UV-Vis spectral analysis of silver was done at room temperature on UV-Vis spec- trophotometer Thermo Genesys UV 10 at resolution of $1 \mathrm{~nm}$.

\subsection{TEM Studies of Silver Nanoparticles}

Transmission electron microscopy (TEM) analysis was prepared by drop-coating Ag nanoparticle solutions onto carbon-coated copper TEM grids. The films on the TEM grids were allowed to stand for $2 \mathrm{~min}$, following which the extra solution was removed using a blotting paper and the grid allowed for drying prior to measurement. TEM measurements were performed on a JEOL Model $1200 \mathrm{EX}$ instrument operated at an accelerating voltage at $120 \mathrm{kV}$.

\subsection{FTIR Analysis of Silver Nanoparticles after Bioreduction}

For Fourier transform infrared (FTIR) spectroscopy measurements, dry powders of the nanoparticles were obtained in the following manner. The Ag nanoparticles synthesized after $24 \mathrm{~h}$ of reaction of the Silver nitrate with the olive seed extract were centrifuged at $10,000 \mathrm{rpm}$ for 15 min, following which the pellet was dispersed in sterile distilled water to get rid of any uncoordinated biological molecules. The process of centrifugation and dispersion in sterile distilled water was repeated three times to ensure better separation of free entities from the metal nanoparticles. The purified pellets were then dried and the powders subjected to FTIR spectroscopy measurement.

\subsection{Antifungal Assay}

The silver nanoparticles in water were tested for their antifungal activity by the agar well diffusion method. The plant pathogenic fungal strain, Aspegillus niger, Aspergillus flavus, Rhizoctonia bataticola, Sclerotium rolfsii, Alternaria macrospora a causative agents of collar rot, root rot, dry rot, stem rot and leaf spot respectively, was 
used for this analysis. This fungal spore suspension was seeded in Czapek-Dox agar plates by the pour plate technique. Three cavities were made using a cork borer (10 $\mathrm{mm}$ diameter) at an equal distance and were filled with the silver nanoparticle solution and then incubated at room temperature for 7 days. The formation of a clear zone (restricted fungal growth) around the cavity is an indication of antifungal activity.

\section{Results and Discussion}

\section{Characterization of Silver Nanoparticles}

It is well known that silver nanoparticles exhibit reddish brown color in aqueous solution due to excitation of surface plasmon vibrations in silver nanoparticles. $\mathrm{Ag}^{2+}$ ions of silver nitrate are found to be reduced to $\mathrm{Ag}$ atoms. It is generally recognized that UV-Vis spectroscopy could be used to examine size and shape controlled nanoparticles in aqueous suspensions. Figure 1 show the UV-Vis spectrum recorded from the reaction medium after 6 hours and gives rise to an absorption band at $419 \mathrm{~nm}$ [9].

To identify the possible biomolecules responsible for the reduction of the $\mathrm{Ag}^{+}$ions and excapping of the bioreduced silver nanoparticles synthesized using olive seed extract, FTIR studies were carried out and the representtative spectrum of the nanoparticles obtained in the present study is presented in Figure 2. Among them, the absorption peak at around 2921, 1562, 1442, $1104 \mathrm{~cm}^{-1}$ can be assigned as absorption peaks. The broad peak obtained at the 2921 region suggests the presence of organic acid groups which support the higher fungicidal efficacy of silver nanoparticles as already mentioned above under reference [8] that olive plant has fungicidal organic acids like Protocatechuic acid, Coumaric acid, Ferulic acid etc. The narrow peaks at 1562 and 1442 regions clearly evince the presence of stretches of $\mathrm{C}=\mathrm{C}-\mathrm{C}$ and

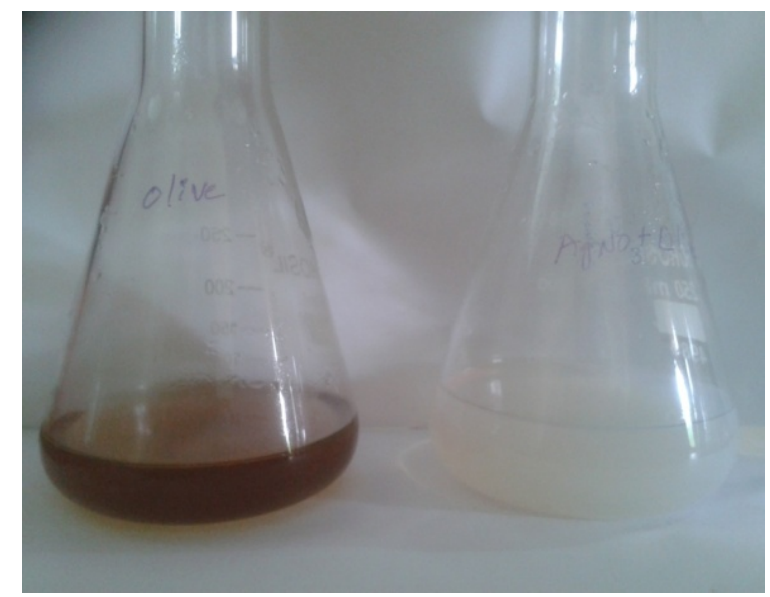

(a)
$\mathrm{C}-\mathrm{H}$ bonds in aromatic rings that are found in Coumaric acid, Ferulic acid etc. The final peak at the 1104 region corroborates the antifungal activity further due to the presence of compounds from olive seed extract that have ether and ester forming $\mathrm{C}=\mathrm{O}$ setups [10-12].

Hence from the above observations, it can be inferred that the silver nanoparticles produced from olive seed extract have higher microbicidal activity due to the presence of active biological compounds from olive seeds. Transmission electron microscopy (TEM) analysis finally confirmed the synthesis of spherical silver nanoparticles in the reaction mixture (Figure 3) which can have a higher surface area to mass ratio. Following the TEM study, a clear indication is obtained regarding the shape and size of the nanoparticles and the silver nanoparticles formed were predominantly of monodisperse nature with their diameter ranging approximately from 2 to $30 \mathrm{~nm}$, which are certainly very much in the size range to increase surface plasmon resonance effect and to impart higher antimicrobial effect.

Diligent observation of various magnifications of TEM images of the synthesized silver nanoparticles clearly define the range of the size from 2 to $20 \mathrm{~nm}$ and the FTIR analyses confirm that the larger size of the nanoparticles might be due to the capping of nanoparticles by biologically active natural acids from olive plants. Silver nanoparticles of a relatively large surface area may easily interact with other particles and increase their antimicrobial efficiency [13]. The synthesized silver NPs consist of spherecal-shaped with irregular contours. The resultant histogram representing the size distribution of the particles was obtained by digital analysis of TEM images containing particles. The distribution of the particle diameters showed as main peak located between 10 and $40 \mathrm{~nm}$ and the sizes ranging from 1 to $50 \mathrm{~nm}$ with an average particles size of $30 \mathrm{~nm}$ (Figure 4).

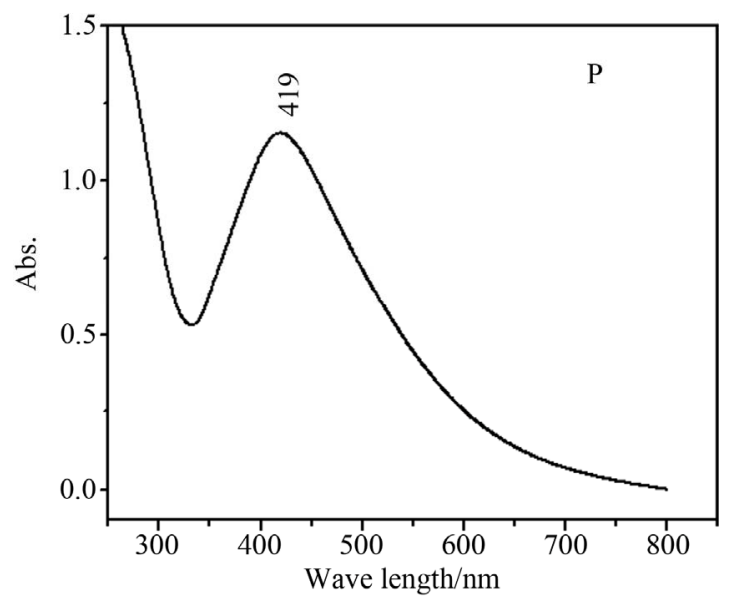

(b)

Figure 1. (a) Silver nanoparticles before and after bioreduction $\left(\mathrm{Ag}^{+}\right.$to $\left.\mathrm{Ag}^{\mathbf{0}}\right)$; (b) $\mathrm{UV}$ absorption spectra of silver nanoparticles after bioreduction by olive seed extract. 


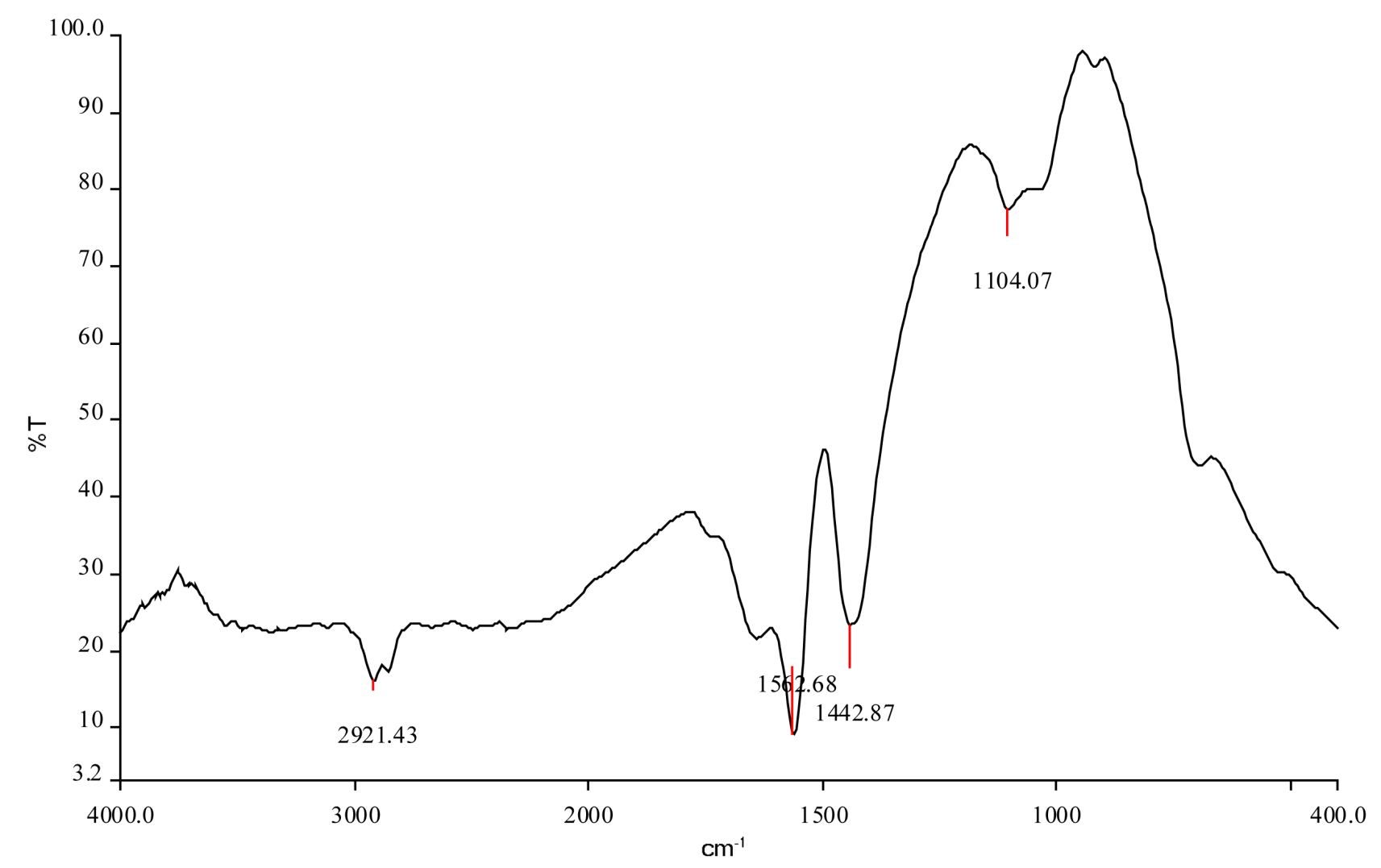

Figure 2. FTIR spectra of Agnps.

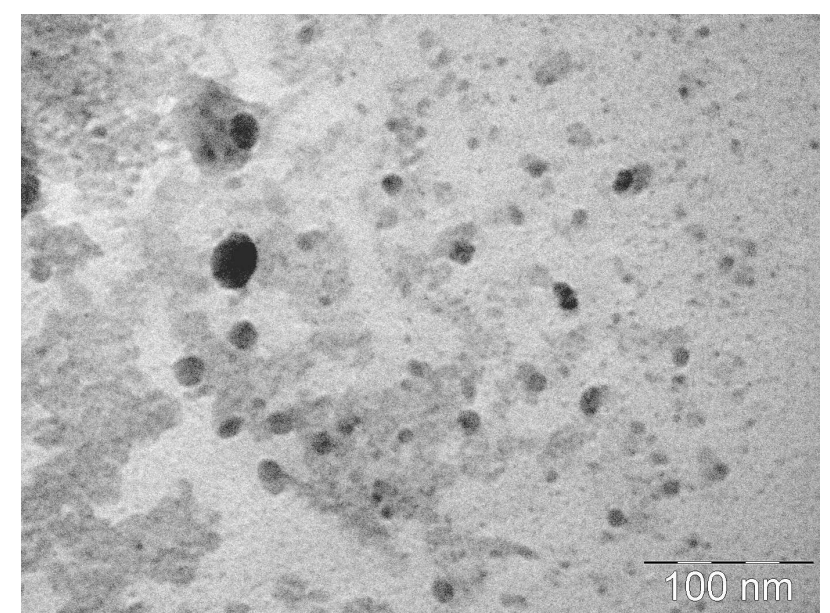

Figure 3. TEM micrographs of Agnps.

Silver is highly antimicrobial and this effect is dependent on superficial contact with enzymatic systems of the respiratory chain by alter DNA synthesis $[14,15]$. Size reduction i.e. the nano-scale particle syntheses involve the increase of surface area for contact, which is an important tool for the effects of silver and reasons to consider silver as superior is its broad antimicrobial activity [16]. So we derive enhanced antimicrobial activity without aesthetic modifications and sticking to the low production cost can be achieved by using silver nanoparti-

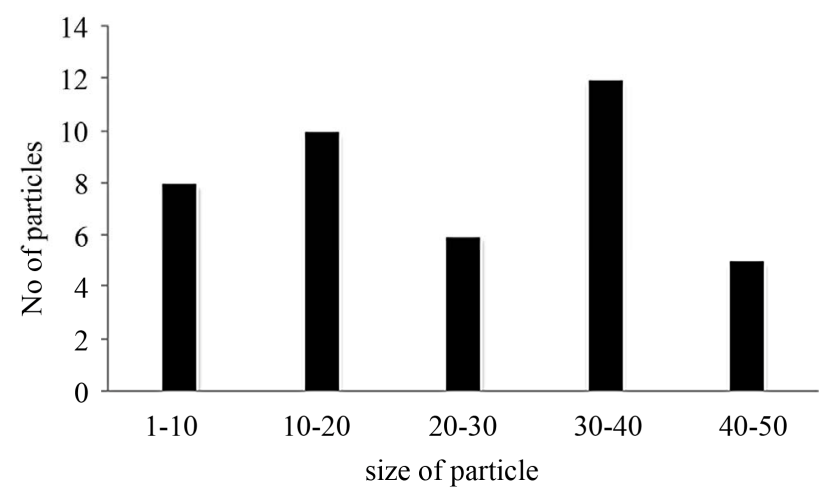

Figure 4. Size distributions of Ag nanoparticles.

cles. Ever growing antibiotic resistant strains of bacteria constantly forces the scientific community to search for and to develop novel antibiotics and many new antibiotics have been introduced in the last decade and unfortunately none of them was successful in combatting the multi-drug resistant strains [17]. As the nanoparticles have delivered and demonstrated effective antimicrobial activities, the development of novel preparations in this field finds attractive alternative to antibiotics. To authenticate, nanoparticles have been examined for their ability to suppress microbial infections in skin [18] and burn wounds [19], and even in preventing bacterial colonization and the results are promising. Reduction in the size 
of the particles of the materials is an efficient and reliable tool for reinforcing their biocompatibility. In fact, nanotechnology helps in overcoming the limitations of size and can change the outlook of the world regarding science [20]. Studies reveal that the antimicrobial activity of the silver particles is due to their positive charge that qualifies them in reacting with the negatively charged proteins on the cell membranes and thus contributing to their antimicrobial activities [21-23]. Many reports have suggested the efficacy of silver nanoparticles of their antimicrobial activities and to mention some as follows. Kim et al. [24] have obtained positive results against $E$. coli and $S$. aureus where as a more profound effect was seen against $E$. coli and comparably a milder effect against S. aureus. Kim et al. [24] also obtained strong antifungal activity for silver nanoparticles against Yeast cells. They further observed a concentration dependant toxicity of silver nanoparticles against bacteria with nanoparticles ranging from $3.3 \mathrm{nM}$ to $6.6 \mathrm{nM}$ [7]. The antifungal effects of silver nanoparticles were estimated against eighteen plant pathogenic fungi that included genera of $P y$ thium, Fusarium, Alternaria, Botrytis, Cladosporium, Corynespora, Cylindrocarpon, Stemphylium etc., by Kim et al. [24]. The antifungal activity of the synthesized nanoparticles has been tested against different fungal plant pathogens namely Aspergillus niger (Collar rot), Aspergillus falvus (Root rot), Rhizoctonia bataticola (Dry rot), Sclerotium rolfsii (Stem rot) and Alternaria macrospora (Leaf spot) using agar well diffusion method. The results were represented in Table 1. The Aspergillus niger was sensitive to the nanoparticles and it showed the zone of inhibition up to $1.6 \mathrm{~cm}$.

The Rhizoctonia bataticola showed lower resistance and the zone of inhibition was measured as $1.3 \mathrm{~cm}$ when compared to the other plant fungal pathogens. This is consistent with previous reports that stated antimicrobial activity of silver was different depending on microbial species.

\section{Conclusion}

The present study demonstrates the ecofriendly nature of the synthesis of silver nanoparticles at a rate on par with

Table 1. Fungicidal activity of silver nanoparticles.

\begin{tabular}{ccccc}
\hline \multirow{2}{*}{ Fungal strains } & \multicolumn{4}{c}{ Zone of inhibition( in cm) } \\
\cline { 2 - 5 } & $25 \mu \mathrm{l}$ & $50 \mu \mathrm{l}$ & $75 \mu \mathrm{l}$ & $100 \mu \mathrm{l}$ \\
\hline Aspergillus niger & 0.5 & 0.8 & 1.2 & 1.6 \\
Aspergillus flavus & 0.3 & 0.6 & 1.0 & 1.4 \\
Rhizoctonia bataticola & 0.4 & 0.5 & 0.9 & 1.3 \\
Sclerotium rolfsii & 0.3 & 0.7 & 1.0 & 1.4 \\
Alternaria macrospora & 0.4 & 0.8 & 1.1 & 1.5 \\
Standard (Clotrimazole antibiotic) & 0.5 & 0.7 & 1.1 & 1.4 \\
\hline
\end{tabular}

chemical synthesis from the plant seed extract. The synthesized nanoparticles were characterized by UV-VIS, FTIR and TEM analysis. The average size of the silver nanoparticles was ranging from 10 to $30 \mathrm{~nm}$. Peak associated with proteins/enzymes on FTIR analysis was appears more likely that the reduction of silver ions and stabilization of synthesized silver nanoparticles is the responsibility of many functional groups, including amines, alcohols, ketenes, aldehydes, and carboxylic acids, that are present in various metabolites such as terpenoids and reducing sugars. The silver nanoparticles thus synthesized are potential enough to kill pathogenic fungi, Aspegillus niger a causative agent of Aspergillosis in human beings.

\section{REFERENCES}

[1] T. L. Riddin, M. Gericke and C. G. Whiteley, "Analysis of the Inter- and Extracellular Formation of Platinum Nanoparticles by Fusarium oxysporum. sp. Lycopersicum Using Surface Response Methodology," Nanotechnology, Vol. 17, No. 14, 2006, pp. 3482-3489. doi:10.1088/0957-4484/17/14/021

[2] K. B. Narayana and N. Sakthivel, "Biological Synthesis of Metal Nanoparticles by Microbes," Advances in Colloids and Interface Science, Vol. 156, No. 1-2, 2010, pp. 1-13. doi:10.1016/j.cis.2010.02.001

[3] S. P. Chandran, M. Chaudhary, R. Pasricha, A. Ahmad and M. Sastry, "Synthesis of Gold Nano Triangles and Silver Nanoparticles Using Aloe Vera Plant Extract," Biotechnology Progress, Vol. 22, No. 2, 2006, pp. 577-583. doi:10.1021/bp0501423

[4] M. Sastry, A. Ahmad, K. M. Islam and R. Kumar, "Biosynthesis of Metal Nanoparticles Using Fungi and Actinomycetes," Current Science, Vol. 85, No. 2, 2003, pp. 162-170.

[5] M. Uchida, T. Yamamoto and A. Taniguchi, "Reaction of Silver Ions and Some Aminoacids," Journal of Antibacterial and Antintifungal Agents, Vol. 31, No. 11, 2003, pp. 695-704.

[6] R. Kumar and H. Münstedt, "Silver Ion Release from Antimicrobial Polyamide/Silver Composites," Biomaterials, Vol. 26, No. 14, 2005, pp. 2081-2088. doi:10.1016/j.biomaterials.2004.05.030

[7] J. S. Kim, E. Kuk, K. N. Yu, J. H. Kim, S. J. Park, H. J. Lee, S. H. Kim, Y. K. Park, Y. H. Hwang, Y. K. Kim, Y. S. Lee, D. H. Jeong and M. H. Cho, "Antimicrobial Effects of Silver Nanoparticles," Nanomedicine, Vol. 3, No. 1, 2007, pp. 95-101. doi:10.1016/j.nano.2006.12.001

[8] K. I. Al-Mughrabi, T. A. Aburjai, G. H. Anfoka and W. Shahrour, "Antifungal Activity of Olive Cake Extracts," Phytopathologia Mediterranea, Vol. 40, No. 3, 2001, pp. 240-244.

[9] S. Shankar, A. Ahmad and M. Sastry, "Geranium Leaf Assisted Biosynthesis of Silver Nanoparticles," Biotechnology Progress, Vol. 19, No. 6, 2003, pp. 1627-1631. doi:10.1021/bp034070w 
[10] V. Bansal, D. Rautaray, A. Bharde, K. Ahire, A. Sanyal, A. Ahmad, et al., "Fungus-Mediated Biosynthesis of Silica and Titania Particles," Journal of Materials Chemistry, Vol. 15, No. 26, 2005, pp. 2583-2589. doi:10.1039/b503008k

[11] J. Huang, Q. Li, D. Sun, Y. Lu, Y. Su, X. Yang, et al., "Biosynthesis of Silver and Gold Nanoparticles by Novel Sundried Cinnamomum Camphora Leaf," Nanotechnology, Vol. 18, No. 10, 2007, Article ID: 105104. doi:10.1088/0957-4484/18/10/105104

[12] R. Sanghi and P. Verma, "Biomimetic Synthesis and Characterisation of Protein Capped Silver Nanoparticles," Bioresource Technology, Vol. 100, No. 1, 2009, pp. 501504. doi:10.1016/j.biortech.2008.05.048

[13] M. Rai, A. Yadav and A. Gade, "Silver Nanoparticles as a New Generation of Antimicrobials," Biotechnology Advances, Vol. 27, No. 1, 2009, pp. 76-83. doi:10.1016/j.biotechadv.2008.09.002

[14] D. W. Brett, "A Discussion of Silver as an Antimicrobial Agent: Alleviating the Confusion," Ostomy Wound Manage, Vol. 52, No. 1, 2006, pp. 34-41.

[15] E. Hidalgo and C. Domínguez, "Study of Cytotoxicity Mechanisms of Silver Nitrate in Human Dermal Fibroblast," Toxicology Letters, Vol. 98, No. 3, 1998, pp. 169179. doi:10.1016/S0378-4274(98)00114-3

[16] P. Spacciapoli, D. Buxton, D. Rothstein and P. Friden, "Antimicrobial Activity of Silver Nitrate against Periodontal Pathogens," Journal of Periodontal Research, Vol. 36, No. 2, 2001, pp. 108-113. doi:10.1034/j.1600-0765.2001.360207.x

[17] J. M. Conlon, J. Kolodziejek and N. Nowotny, "Antimicrobial Peptides from Ranid Frogs: Taxonomic and Phylogenetic Markers and a Potential Source of New Therapeutic Agents," Biochimica et Biophysica Acta, Vol. 1696, No. 1, 2004, pp. 1-14. doi:10.1016/j.bbapap.2003.09.004

[18] J. E. Paddle-Ledinek, Z. Nasa and H. J. Cleland, "Effect of Different Wound Dressings on Cell Viability and Proliferation," Plastic and Reconstructive Surgery, Vol. 117, No. 7, 2006, pp. 110S-118S. doi:10.1097/01.prs.0000225439.39352.ce

[19] E. Ulkur, O. Oncul, H. Karagoz, E. Yeniz and B. Celikoz, "Comparison of Silver-Coated Dressing (Acticoat), Chlorhexidine Acetate $0.5 \%$ (Bacti-Grass), and Fusidic Acid 2\% (Fucidin) for Topical Antibacterial Effect in Methicillin-Resistant Staphylococci-Contaminated, Full-Skin Thickness Rat Burn Wounds,” Burns, Vol. 31, No. 7, 2005, pp. 874-877. doi:10.1016/i.burns.2005.05.002

[20] C. A. Mirkin and T. A. Taton, "Semiconductors Meet Biology," Nature, Vol. 405, No. 6787, 2000, pp. 626-627. doi:10.1038/35015190

[21] T. Hamouda, A. Myc, B. Donovan, A. Shih, J. D. Reuter and J. R. Baker, "A Novel Surfactant Nanoemulsion with a Unique Non-Irritant Topical Antimicrobial Activity against Bacteria, Enveloped Viruses and Fungi," Microbiological Research, Vol. 156, No. 1, 2000, pp. 1-7. doi:10.1078/0944-5013-00069

[22] P. Dibrov, J. Dzioba, K. K. Gosink and C. C. Häse, "Chemiosmotic Mechanism of Antimicrobial Activity of $\mathrm{Ag}^{(+)}$in Vibrio Cholera," Antimicrob Agents Chemother, Vol. 46, No. 8, 2002, pp. 2668-2670. doi:10.1128/AAC.46.8.2668-2670.2002

[23] I. Dragieva, S. Stoeva, P. Stoimenov, E. Pavlikianov and K. Klabunde, "Complex Formation in Solutions for Chemical Synthesis of Nanoscaled Particles Prepared by Borohydride Reduction Process," Nanostructured Materials, Vol. 12, No. 1, 1999, pp. 267-270. doi:10.1016/S0965-9773(99)00114-2

[24] S. W. Kim, J. H. Jung, K. Lamsal, J. S. Min and Y. S. Lee, "Antifungal Effects of Silver Nanoparticles (AgNPs) against Various Plant Pathogenic Fungi," Mycobiology, Vol. 40, No. 1, 2012, pp. 53-58. doi:10.5941/MYCO.2012.40.1.053 